Article

\title{
Addition of Fish Oil to Cream Cheese Affects Lipid Oxidation, Sensory Stability and Microstructure
}

\author{
Anna Frisenfeldt Horn ${ }^{1}$, Ditte Green-Petersen ${ }^{1}$, Nina Skall Nielsen ${ }^{1}$, Ulf Andersen ${ }^{2}$, \\ Grethe Hyldig ${ }^{1}$, Louise Helene Søgaard Jensen ${ }^{3,4}$, Andy Horsewell ${ }^{3,4}$ and Charlotte Jacobsen ${ }^{1, *}$ \\ 1 Division of Industrial Food Research, National Food Institute, Technical University of Denmark, \\ Søltofts Plads, Building 221, DK-2800 Kgs. Lyngby, Denmark; \\ E-Mails: afho@food.dtu.dk (A.F.H.); digr@food.dtu.dk (D.G.-P.);nsni@food.dtu.dk (N.S.N.); \\ grhy@food.dtu.dk (G.H.) \\ 2 Arla Foods amba, Arla Strategic Innovation Centre, DK-8220, Brabrand, Denmark; \\ E-Mail: ulf.andersen@arlafoods.com
}

3 Department of Mechanical Engineering, Technical University of Denmark, Niels Koppels Allé 404, DK-2800 Kgs. Lyngby, Denmark; E-Mails: losj@mek.dtu.dk (L.H.S.J.); anho@mek.dtu.dk (A.H.)

4 Center for Electron Nanoscopy, Technical University of Denmark, Fysikvej, building 307, DK-2800 Kgs. Lyngby, Denmark

* Author to whom correspondence should be addressed; E-Mail: chja@food.dtu.dk; Tel.: +45-4525-2559; Fax: +45-4588-4774.

Received: 12 September 2012; in revised form: 15 October 2012 / Accepted: 16 October 2012 / Published: 12 November 2012

\begin{abstract}
The objective of this study was to investigate the differences in the oxidative stability during storage of fish oil enriched cream cheeses when fish oil was added either as neat oil or pre-emulsified oil with sodium caseinate, whey protein isolate, or a combination of milk proteins and phospholipids as emulsifier. Results showed that the addition of fish oil decreased the oxidative stability of cream cheeses regardless of the addition method, especially when the cheese was stored longer than five weeks. The oxidative stability of fish oil enriched cream cheeses was highest when fish oil was added as neat oil or in a delivery emulsion prepared with a combination of milk proteins and phospholipids. Adding the fish oil in a delivery emulsion prepared with whey protein or caseinate resulted in a less oxidative stable product. It was furthermore shown that the microstructure of the cream cheeses was affected by fish oil addition, and it was suggested that the change in microstructure was partly responsible for the oxidative stability of the cream cheeses.
\end{abstract}


Keywords: omega-3 fatty acids; delivery emulsion; whey protein isolate; sodium caseinate; milk phospholipids; peroxide value; volatile oxidation products; sensory evaluation; confocal laser scanning microscopy

\section{Introduction}

Both epidemiological and intervention studies have shown that long chain polyunsaturated omega-3 fatty acids possess a wide range of health beneficial effects [1]. For this reason, an increasing interest in substituting the original fat in some food products with fish oil that is rich in eicosapentaenoic acid (EPA, 20:5n-3) and docosahexaenoic acid (DHA, 22:6n-3) has developed. However, a major concern in fish oil enriched foods is lipid oxidation and the development of volatile oxidation products resulting in undesirable off-flavors in the fish oil enriched food product.

Dairy products are in general considered healthy food products. Moreover, they have a natural content of the potentially antioxidative milk proteins and are thus considered good vehicles for fish oil addition. However, studies have shown that various fish oil enriched dairy products might differ to a great extent in their oxidative stability [2,3]. To the authors' knowledge, only one study has previously been reported on fish oil enriched cream cheese, and this study only evaluated the sensory performance of the cheeses [4]. It was observed that it was possible to add $15 \mathrm{~g}$ fish oil per $\mathrm{kg}$ spreadable fresh cheese (Philadelphia type) before reaching a level where the sensory quality was significantly impacted. In comparison, it was possible to add $3 \mathrm{~g}$ fish oil per $\mathrm{kg}$ non-flavored semi-solid processed fresh low-fat cheese (110 g fat $/ \mathrm{kg})$ and $40 \mathrm{~g}$ fish oil per $\mathrm{kg}$ non-flavored processed cheese (320 g fat $/ \mathrm{kg}$ ). The spreadable fresh cheese with $15 \mathrm{~g}$ fish oil $/ \mathrm{kg}$ could be stored for up to 5 weeks before the sensory quality decreased. Since the shelf life of a cream cheese is usually more than 20 weeks the above-mentioned results indicate that the addition of fish oil has to be improved in order to avoid lipid oxidation and increase shelf life. One strategy to protect the unsaturated fatty acids could be to incorporate them in an emulsion (a delivery emulsion) prior to their addition to the food product.

This approach was evaluated in processed cheese, and it was observed that the use of a delivery emulsion prepared with a milk protein complex (casein and whey protein) as emulsifier increased the oxidative stability throughout storage as compared to the addition of neat oil [3]. However, despite the better oxidative stability, the sensory perception of the cheese was still different from the control upon storage when fish oil was added in a concentration of $30 \mathrm{~g}$ or more per $\mathrm{kg}$ cheese (corresponding to approximately $89 \mathrm{mg}$ fish oil in a serving size of $30 \mathrm{~g}$ cheese). Thus, improvements of the delivery emulsion used could be advantageous, as could a better understanding of lipid oxidation in these types of food products.

The aim of this study was to compare lipid oxidation in fish oil enriched cream cheese upon storage, when $1.3 \%$ fish oil was added as a $70 \%$ fish oil-in-water delivery emulsion or as neat oil. This amount of fish oil makes it possible to claim "High in omega-3" according to EU Commission Regulation No 116/2010 [5]. Delivery emulsions were prepared with either sodium caseinate, whey protein isolate or an emulsifier with a combination of milk proteins and milk phospholipids. Fish oil enriched cream cheese was compared to cream cheese without fish oil. Lipid oxidation was followed by the 
development in peroxide value, concentration of volatile secondary oxidation products and sensory performance over 20 weeks of storage. The microstructure of the cream cheeses was furthermore imaged by confocal laser scanning microscopy.

\section{Materials and Methods}

\subsection{Materials}

Raw cream cheese was provided by Arla Foods Holstebro Flødeost (Holstebro, Denmark). Sodium caseinate (Miprodan ${ }^{\circledR}$ 30), whey protein isolate (Lacprodan ${ }^{\circledR}$ DI-9224), and milk phospholipid/milk protein (Lacprodan ${ }^{\circledR}$ PL-20) were kindly donated by Arla Foods Ingredients amba (Viby J, Denmark). Cod liver oil was provided by Maritex A/S, subsidiary of TINE, BA (Sortland, Norway). The oil was stored at $-40{ }^{\circ} \mathrm{C}$ until use. The initial PV in the fish oil and the content of tocopherol compounds were determined as described in section 2.7.1 and 2.6, respectively, and results are given in Table 2.

\section{2. $\quad$ Preparation of $70 \%$ Fish Oil-in-Water Emulsions}

Delivery emulsions were prepared as described by Horn et al. [6] in a Stephan Universal mixer (Stephan, UMC5, 1995, Hameln, Germany) with water instead of buffer as the continuous phase. Three emulsions were prepared (1000 g of each) with 70.0\% fish oil, $27.2 \%$ distilled water and $2.8 \%$ emulsifier. The emulsifiers were sodium caseinate, whey protein isolate or an emulsifier with a combination of milk phospholipids (approximately 20\%) and milk proteins (approximately 50\%). The pressure was reduced in the mixer during production to minimize air bubbles in the emulsions. Emulsions were kept at approximately $5{ }^{\circ} \mathrm{C}$ until production of the fish oil enriched cream cheese. The emulsions are named Em_CAS (emulsion with sodium caseinate), Em_WPI (emulsion with whey protein isolate) and Em_MPL20 (emulsion with a combination of milk phospholipids and milk proteins) according to the emulsifier used for their production.

\subsection{Production of Fish Oil Enriched Cream Cheese}

Enriched cream cheeses (batches of $25 \mathrm{~kg}$ ) were prepared in a pilot plant at Arla Foods amba, Arla Strategic Innovation Centre (Brabrand, Denmark). Raw cheese was taken from an intermediate step of the production of cream cheese (50+). Raw cheese was weighted off and poured into a $50 \mathrm{~L}$ Stephan Universal mixer (Stephan, UMMISK 40E-GNI, 1979, Hameln, Germany), and held under vacuum for $30 \mathrm{~s}$ before being pasteurized by heating to $72{ }^{\circ} \mathrm{C}$ at $1500 \mathrm{rpm}$. Hereafter, either neat oil or one of the delivery emulsions was added to the heated cheese $(1.3 \%$ fish oil $\mathrm{w} / \mathrm{w})$, and the cheese was stirred for $5 \mathrm{~min}$. Then the cheese was held under vacuum once more for $60 \mathrm{~s}$, before it was moved to a high pressure homogenizer (Invensys APV, R18-38, Silkeborg, Denmark), where it was homogenized at $65{ }^{\circ} \mathrm{C} / 175$ bar. A reference cheese was prepared by a similar heating and homogenization procedure, but without addition of fish oil. All cream cheeses were tapped directly into $300 \mathrm{~g}$ white plastic containers while still warm, and sealed before cooling to approximately $5{ }^{\circ} \mathrm{C}$. 


\subsection{Storage and Sampling}

Both the fish oil enriched cream cheeses and the reference cheese were stored at $4.6 \pm 0.4{ }^{\circ} \mathrm{C}$ in white plastic containers for 20 weeks in the dark. Samples were taken at week 0, 2, 5, 10, 15 and 20 for lipid oxidation analyses. Furthermore, samples of the three delivery emulsions and the neat oil were taken for PV determination at the day of production. The $\mathrm{pH}$ value was determined in both the three delivery emulsions and the five cream cheese samples at day 0 or 1 and week 20, respectively. The neat oil and the five cream cheese samples were furthermore characterized by fatty acid compositions and their contents of tocopherols.

\subsection{Characterization of the Delivery Emulsions and Cream Cheeses}

\subsubsection{Droplet Size and $\mathrm{pH}$}

Droplet size distributions were determined in the delivery emulsions by laser diffraction on a Mastersizer 2000 (Malvern Instruments, Ltd., Worcestershire, UK). The emulsions were pretreated according to the method described by Let et al. (2007) [2]. Emulsion (1 g) was dissolved in $5 \mathrm{~g}$ SDS buffer ( $\left.10 \mathrm{mM} \mathrm{NaH}_{2} \mathrm{PO}_{4}, 5 \mathrm{mM} \mathrm{SDS}\right)$, mixed for 30 seconds and then sonicated for $15 \mathrm{~min}$ in a water bath at $0{ }^{\circ} \mathrm{C}$. Droplets of the pretreated emulsions were diluted in recirculating water (3000 rpm), reaching an obscuration of $12 \%-15 \%$. The refractive indices of sunflower (1.469) and water (1.330) were used as particle and dispersant, respectively.

In both delivery emulsions and cream cheeses, the $\mathrm{pH}$ was determined in a suspension of the emulsion in distilled water $(1: 1)$.

\subsubsection{Confocal Laser Scanning Microscopy}

The cream cheeses were subjected to imaging by confocal microscopy. Prior to that, samples were stained with Fluorescein isothiocyanate for the proteins and Nile red for the oil. Microscopy was performed on a Leica TCS SP II (Leica Microsystems GmbH, Heidelberg, Germany) inverted vertically, at room temperature with a 100x oil immersion objective.

\subsection{Lipid Extraction, and Analyses of Fatty Acid Compositions and Tocopherols}

Lipids were extracted from all samples using a modified form of the method described by Bligh and Dyer (1959) [7] with a reduced amount of solvent (30.0 mL methanol and chloroform, 1:1). For lipid extractions on the delivery emulsions $5 \mathrm{~g}$ sample was used, and on the fish oil enriched cream cheeses 10 g sample was used.

Fatty acid compositions were determined directly on the neat oil by fatty acid methylation [8] followed by separation through gas chromatography (HP 7890 A, Hewlett Packard, Palo Alto, CA, USA; Column: DB-WAX, $10 \mathrm{~m} \times 0.1 \mathrm{~mm} \times 0.1 \mu \mathrm{m}$ ) according to the official AOCS method [9].

The content of tocopherol was determined by HPLC (Agilent 1100 Series; Column: Waters Spherisorb $3 \mu \mathrm{m}$ Silica; $4.6 \times 150 \mathrm{~mm}$ ), by injecting each lipid extract twice. Tocopherols were analyzed according to the official AOCS method [10]. 


\subsection{Measurements of Lipid Oxidation}

\subsubsection{Primary Oxidation Products-Peroxide Values}

Peroxide values (PV) were determined on the lipid extracts (prepared as described in section 2.6), or directly on the neat oil, by colorimetric determination of iron thiocyanate at $500 \mathrm{~nm}$ as described by Shantha and Decker (1994) [11].

\subsubsection{Secondary Oxidation Products_-Dynamic Headspace GC-MS}

Cream cheese $(5-8 \mathrm{~g})$ was added $10 \mathrm{~mL}$ distilled water and purged with nitrogen $(150 \mathrm{~mL} / \mathrm{min})$ for $30 \mathrm{~min}$ at $45{ }^{\circ} \mathrm{C}$ and trapped on Tenax GR tubes. Subsequently, water was removed by purging the tube in the opposite direction with nitrogen for $20 \mathrm{~min}(50 \mathrm{~mL} / \mathrm{min})$. The volatiles were desorbed again by heat $\left(200{ }^{\circ} \mathrm{C}\right)$ in an Automatic Thermal Desorber (ATD-400, Perkin Elmer, Norwalk, CN), cryofocused on a cold trap $\left(-28^{\circ} \mathrm{C}\right)$, released again $\left(220^{\circ} \mathrm{C}\right)$, and led to a gas chromatograph (HP 5890 Series, Hewlett Packard, Palo Alto, CA, USA; Column: DB-1701, $30 \mathrm{~m} \times 0.25 \mathrm{~mm} \times 1.0 \mu \mathrm{m}$; J\&W Scientific, CA, USA) with mass spectrometrical detection (HP 5972 inert mass-selective detector, Agilent Technologies, USA). The oven program in the gas chromatograph had an initial temperature of $45{ }^{\circ} \mathrm{C}$ for $5 \mathrm{~min}$, increasing with $1.5{ }^{\circ} \mathrm{C} / \mathrm{min}$ until $55{ }^{\circ} \mathrm{C}$, with $2.5{ }^{\circ} \mathrm{C} / \mathrm{min}$ until $90{ }^{\circ} \mathrm{C}$ and with $12.0^{\circ} \mathrm{C} / \mathrm{min}$ until $220^{\circ} \mathrm{C}$, where the temperature was kept steady for $4 \mathrm{~min}$. The individual compounds were analyzed by mass-spectrometry (Electron ionization mode, $70 \mathrm{eV}$; mass to charge ratios between 30 and 250). From a comparison of chromatograms from non-oxidized and oxidized samples, the following volatiles were selected for quantification: butanal, 2-ethylfuran, $t$-2-butenal, pentanal, 1-penten-3-ol, $t$-2-pentenal, 1-pentanol, hexanal, $t$-2-hexenal, heptanal, 2-pentylfuran, $t$-2-heptenal, $t, t-2,4$-heptadienal. Calibration curves were made by dissolving the compounds in ethanol (96\%) followed by the addition of an amount of this mix corresponding to 3-1000 ng of the compounds to $8 \mathrm{~g}$ of the reference cheese. Volatiles were collected and analyzed similarly as in the cream cheese samples. Measurements were made in triplicate on each sample. Three of the volatiles could not be extracted completely from the chromatograms (1-penten-3-ol, $t$-2-pentenal and heptanal) due to overlapping compounds and were thus later taken out. Calibration curves were parallel shifted in order to obtain positive values, thus the concentrations of volatiles are not given as exact values.

\subsection{Sensory Evaluation}

Sensory evaluation was conducted by a panel consisting of twelve assessors, eight females and four males. The assessors were tested and trained in descriptive sensory analysis according to ISO standards $[12,13]$. Before sensory profiling, a vocabulary of descriptors for odor, appearance, flavor and texture was developed. The descriptors were evaluated on an unstructured $15 \mathrm{~cm}$ scale anchored $1.5 \mathrm{~cm}$ from both ends.

Sensory evaluation was performed five times during the storage period of 20 weeks. The evaluations were performed after 2, 5, 10, 15 and 20 weeks of storage. Due to the long time duration in between evaluations a two-hour training session was held two days before each evaluation was conducted. 
All samples were served individually in closed Petri dishes and the lids were marked with a three-digit code. Each Petri dish contained two separately placed portions of the same cream cheese sample. One of the portions was used for evaluating flavor and texture as perceived in the mouth (pasty, melt down and fatty), while the other portion was used for evaluating the texture (firmness and spreadability) and appearance directly in the Petri dish. All samples were evaluated in duplicates and in randomized order. Evaluations were performed according to ISO standard 8589 [14] in separated booths under normal daylight. To clean the mouth between samples, the assessors used water and peeled cucumber, where the soft center with the pips was removed. Between eight and twelve assessors participated in each evaluation. Data were collected using the computer system FIZZ (Network Version 2.0, Biosystems, Couternon, France).

\subsection{Statistical Analyses}

All chemical data were analyzed by one- or two-way analysis of variance with Bonferronis multiple comparison test as posttest (GraphPad Prism, version 4.03, GraphPad Software Inc., La Jolla, CA, USA). All references to significant differences $(p<0.05)$ between samples or between sampling time points, are based on this statistical analysis of data.

Results from sensory profiling were corrected for level effect by the method of Thybo and Martens (2000) [15]. After level correction a principal component analysis (PCA) on mean values was performed to study the differences between the different types of cream cheese samples and storage times. The correction for level effect and PCA were calculated using The Unscrambler ${ }^{\circledR} 9.1$ (CAMO, Trondheim, Norway).

\section{Results}

\subsection{Characterization of the Neat Oil and Delivery Emulsions}

The fatty acid composition of the fish oil used for the production of delivery emulsions and fish oil enriched cream cheese, showed that it contained approximately $14 \% 48 \%$ and $29 \%$ saturated fatty acids, monounsaturated and polyunsaturated fatty acids, respectively (Table 1). Approximately $27 \%$ of the total fatty acids were n-3 fatty acids. The fish oil had an initial PV of 0.1 meq peroxides $/ \mathrm{kg}$ oil (Table 2). The contents of tocopherols were $207 \mu \mathrm{g} / \mathrm{g}$ oil and $100 \mu \mathrm{g} / \mathrm{g}$ oil of $\alpha$ - and $\gamma$-tocopherol, respectively (Table 2).

The PV in the delivery emulsions were all $\leq 0.3$ meq lipid hydroperoxides $/ \mathrm{kg}$ oil (Table 2 ). The $\mathrm{pH}$ values of the delivery emulsions were 6.8-7.3. The droplet size distributions for all three delivery emulsions were monomodal, but Em_MPL20 had a wider distribution than Em_CAS and Em_WPI (data not shown). The mean oil droplet sizes of the delivery emulsions are given in Table 2. All delivery emulsions were highly viscous with a consistency as yoghurt, and similar emulsions have previously been shown to be physically stable for up to 42 days [6]. 
Table 1. Fatty acid composition of the fish oil determined by fatty acid methylation followed by separation through gas chromatography. Fatty acids are given in area\%.

\begin{tabular}{lclclc}
\hline SFA & & MUFA & & PUFA & \\
\hline Fatty acid & Av [\%] & Fatty acid & Av [\%] & Fatty acid & Av [\%] \\
\hline $14: 0$ & 3.0 & $16: 1(\mathrm{n}-6)$ & 8.2 & $18: 2(\mathrm{n}-6)$ & 1.8 \\
$16: 0$ & 8.9 & $18: 1(\mathrm{n}-9)$ & 16.0 & $18: 3(\mathrm{n}-3)$ & 0.8 \\
$18: 0$ & 1.9 & $18: 1(\mathrm{n}-7)$ & 5.2 & $18: 4(\mathrm{n}-3)$ & 3.5 \\
& & $20: 1(\mathrm{n}-9+\mathrm{n}-11)$ & 11.6 & $20: 4(\mathrm{n}-3)$ & 0.7 \\
& & $22: 1(\mathrm{n}-11)$ & 6.1 & $20: 5(\mathrm{n}-3)$ & 9.3 \\
& & $22: 1(\mathrm{n}-9)$ & 0.8 & $22: 5(\mathrm{n}-3)$ & 1.1 \\
& & & $22: 6(\mathrm{n}-3)$ & 11.6 \\
\hline Total & $\mathbf{1 3 . 8}$ & Total & $\mathbf{4 8 . 3}$ & Total & $\mathbf{2 9 . 4}$ \\
\hline & & & & Total $\mathbf{n - 3}$ & $\mathbf{2 6 . 6}$ \\
\hline
\end{tabular}

Only fatty acids in a concentration $>0.5 \%$ are given by name; All standard deviations are $<0.1 \%$; SFA: Saturated fatty acids; MUFA: Monounsaturated fatty acids; PUFA: Polyunsaturated fatty acids; Av: Average.

Table 2. Data on fish oil and delivery emulsions used for production of the cream cheese samples.

\begin{tabular}{lcccc}
\hline & Fish oil & Em_CAS & Em_WPI & Em_MPL20 \\
\hline Emulsifier type & -- & CAS & WPI & MPL20 \\
pH & -- & 7.1 & 6.8 & 7.3 \\
PV [meq peroxides/kg oil] & $0.1 \pm 0.0$ & $0.2 \pm 0.0$ & $0.1 \pm 0.0$ & $0.3 \pm 0.0$ \\
Droplet size, D3,2 [ $\boldsymbol{\mu m}]$ & -- & 8.3 & 21.2 & 12.6 \\
$\boldsymbol{\alpha}$-tocopherol $[\boldsymbol{\mu g} / \mathbf{g}$ oil] & $207 \pm 16$ & -- & -- & -- \\
$\boldsymbol{\gamma}$-tocopherol $[\boldsymbol{\mu g} / \mathbf{g}$ oil] & $100 \pm 1$ & -- & -- & -- \\
\hline
\end{tabular}

--: Not determined. CAS: Sodium caseinate; WPI: Whey protein isolate; MPL20: A commercially available emulsifier with a combination of milk proteins and milk phospholipids.

\subsection{Characterization of the Cream Cheeses}

The $\mathrm{pH}$ of the cream cheeses was 4.7-4.8 throughout the 20 weeks of storage (Table 3 ). From the microscopic imaging it was observed that the reference cheese and the cheese added fish oil as neat oil had relatively large unprotected lipid droplets (colored red in Figure 1), whereas the three cream cheeses with added fish oil through delivery emulsions had far fewer unprotected oil droplets. Particularly MPL20 could be distinguished from the other samples since more of the lipid (including the milk lipid) was hidden within the protein structure. This observation was confirmed by freeze-fracture cryo-scanning electron microscopy (included as supplementary material). 
Table 3. $\mathrm{pH}$ and tocopherol contents in cream cheeses at week 0 and week 20 of storage. Letters indicate significant differences $(P<0.05)$.

\begin{tabular}{|c|c|c|c|c|c|c|}
\hline \multirow[t]{2}{*}{ Cream cheese sample } & \multicolumn{2}{|c|}{ pH } & \multicolumn{2}{|c|}{$\alpha$-tocopherol [ $\mu \mathrm{g} / \mathrm{g}$ oil] } & \multicolumn{2}{|c|}{$\gamma$-tocopherol $[\mu \mathrm{g} / \mathrm{g}$ oil] } \\
\hline & Week 0 & Week 20 & Week 0 & Week 20 & Week 0 & Week 20 \\
\hline CAS & 4.8 & 4.7 & $26 \pm 5 b$ & $12 \pm 0 \mathrm{ab}$ & $8 \pm 1 b$ & $3 \pm 0 \mathrm{~b}$ \\
\hline WPI & 4.8 & 4.7 & $35 \pm 1 c$ & $11 \pm 0 \mathrm{a}$ & $10 \pm 0 \mathrm{~d}$ & $4 \pm 0 c$ \\
\hline MPL20 & 4.8 & 4.7 & $30 \pm 3 b c$ & $8 \pm 0 \mathrm{a}$ & $9 \pm 0 c$ & $4 \pm 0 c$ \\
\hline FO & 4.8 & 4.7 & $31 \pm 1 b c$ & $8 \pm 1 \mathrm{a}$ & $9 \pm 0 c$ & $4 \pm 0 c$ \\
\hline REF & 4.8 & 4.7 & $16 \pm 1 \mathrm{a}$ & $17 \pm 0 \mathrm{~b}$ & $1 \pm 0 \mathrm{a}$ & $0 \pm 0 \mathrm{a}$ \\
\hline
\end{tabular}

CAS, WPI and MPL20: Cream cheeses with fish oil added as delivery emulsion prepared with sodium caseinate, whey protein isolate and an emulsifier with a combination of milk proteins and phospholipids, respectively. FO: Cream cheese with fish oil added as neat oil. REF: Cream cheese without fish oil added.

Figure 1. Confocal micrographs of the cream cheeses prepared without fish oil added (REF), with fish oil added as neat oil (FO) or fish oil added in a delivery emulsion emulsified by different emulsifiers (MPL20, WPI and CAS). Lipids are stained red, proteins green.

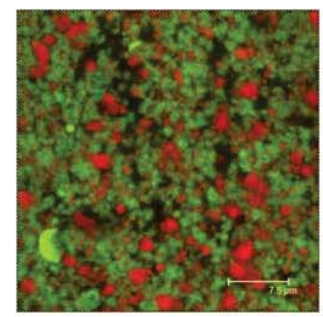

REF

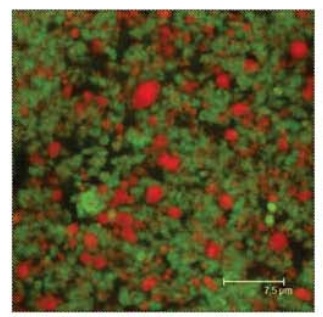

$\mathrm{FO}$

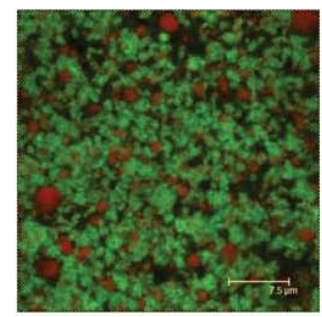

MPL20

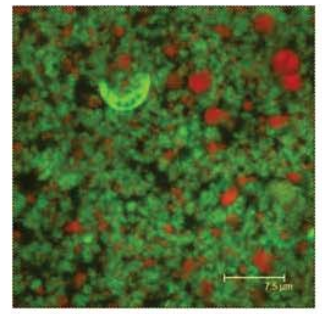

WPI

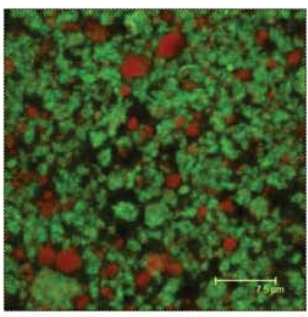

CAS

\subsubsection{Contents of Tocopherol}

The content of $\alpha$-tocopherol was $16 \mu \mathrm{g} / \mathrm{g}$ oil in the reference cream cheese at day 0 as compared to 26-31 $\mu \mathrm{g} / \mathrm{g}$ oil in the cream cheeses with fish oil (Table 3). Similarly, the content of $\gamma$-tocopherol was significantly lower in the reference cream cheese than in the other cream cheeses. The higher content of tocopherols in the cream cheeses with fish oil was due to the endogenous tocopherols in the fish oil itself (Table 2). The content of both $\alpha$ - and $\gamma$-tocopherol were stable in the reference sample during the 20 weeks of storage (Table 3). However, a significant decrease was observed in the contents of both tocopherols for the four samples enriched with fish oil. The content of $\alpha$-tocopherol decreased to $8-12 \mu \mathrm{g} / \mathrm{g}$ oil at week 20 and the content of $\gamma$-tocopherol to $3-4 \mu \mathrm{g} / \mathrm{g}$ oil. The decrease in $\alpha$-tocopherols was lowest in CAS, $14 \mu \mathrm{g} / \mathrm{g}$ oil, compared to $22-24 \mu \mathrm{g} / \mathrm{g}$ oil in the other three cream cheeses with fish oil. 


\subsection{Lipid Oxidation in Emulsions}

\subsubsection{Peroxide Values}

PV were not significantly different between samples at week 0 . However, PV in all samples except the reference increased significantly during storage, and a significant increase was already observed between week 0 and 2 (Figure 2). When comparing samples with fish oil, it was found that these samples did not differ significantly until week 10. At week 10 the sample with MPL20 had a significantly lower PV than the other samples, and WPI a significantly higher PV. However, between week 10 and 20, PV in CAS and WPI did not increase whereas it increased significantly in MPL20 and FO. Thus, at week 20, the rank order was $\mathrm{REF}^{\mathrm{a}}<\mathrm{WPI}^{\mathrm{b}}=\mathrm{CAS}^{\mathrm{b}}<\mathrm{FO}^{\mathrm{c}}=\mathrm{MPL} 20^{\mathrm{c}}$.

Figure 2. Peroxide values in cream cheeses with fish oil added as neat oil (FO), or in a delivery emulsion prepared with different emulsifiers (CAS, WPI or MPL20) compared to a reference cheese without fish oil (REF) during storage for 20 weeks.

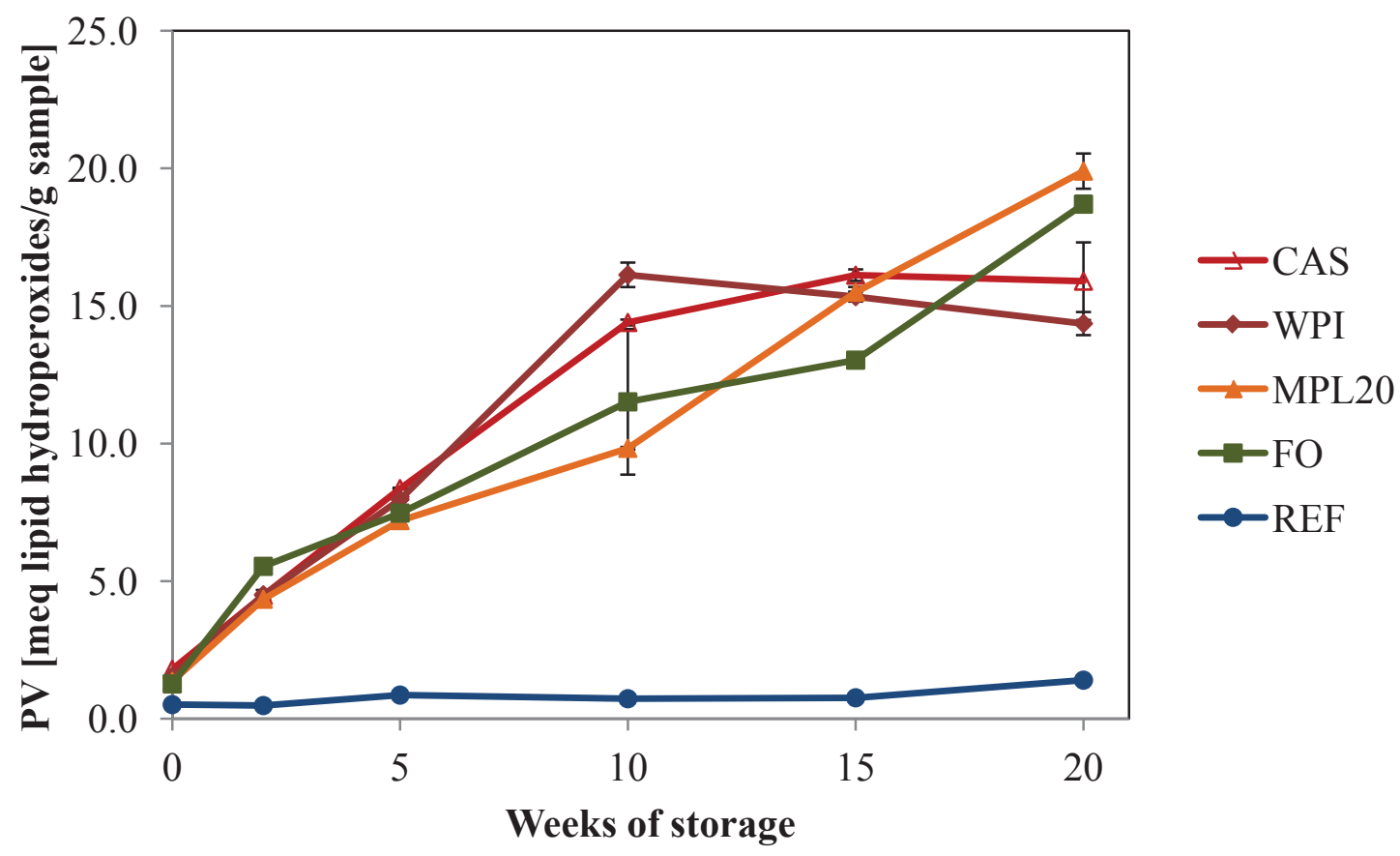

\subsubsection{Secondary Volatile Oxidation Products}

The volatiles pentanal and hexanal stemming from oxidation of $n-6$, and, most important in this context, a wide range of volatiles stemming from oxidation of n-3 fatty acids, such as 2-ethylfurane, $t$-2-butenal, $t$-2-hexenal, and $t, t$-2,4-heptadienal were quantified. The volatiles could be grouped in volatiles that increased significantly in all cream cheese samples during storage (Group 1: pentanal, 1-pentanol, hexanal and 2-pentylfurane, represented by hexanal in Figure 3) and volatiles that only increased in the samples with fish oil added (Group 2: butanal, 2-ethylfurane, $t$-2-butenal, $t$-2-hexenal, $t$-2-heptenal and $t, t-2,4$-heptadienal, represented by $t$-2-hexenal in Figure 4).

As an example of group 1 volatiles, hexanal is presented in Figure 3. It was observed that the concentration of hexanal as well as concentrations of pentanal, 1-pentanol and 2-pentylfurane 
increased significantly in the reference cream cheese between week 0 and 20 . The presence of fish oil in the other cream cheeses increased the rate at which these volatiles were produced, and, at week 20 , all four volatiles were present in significantly higher concentrations in the cream cheeses with fish oil compared to the reference cream cheese. Interestingly, concentrations of these volatiles did not increase significantly in the MPL20 sample until between week 10 and 15 or between week 15 and 20. In comparison, CAS had significantly increased concentration of pentanal already between week 2 and 5 and increased concentrations of hexanal and 2-pentylfurane between week 5 and 10. Concentrations of both hexanal and 2-pentylfuran increased significantly in the sample with fish oil added as neat oil (FO) between week 2 and 5.

Figure 3. The development in hexanal concentrations (ng/g sample) in cream cheeses with fish oil added as neat oil (FO), or in a delivery emulsion prepared with different emulsifiers (CAS, WPI or MPL20) compared to a reference cheese without fish oil (REF) during storage for 20 weeks.

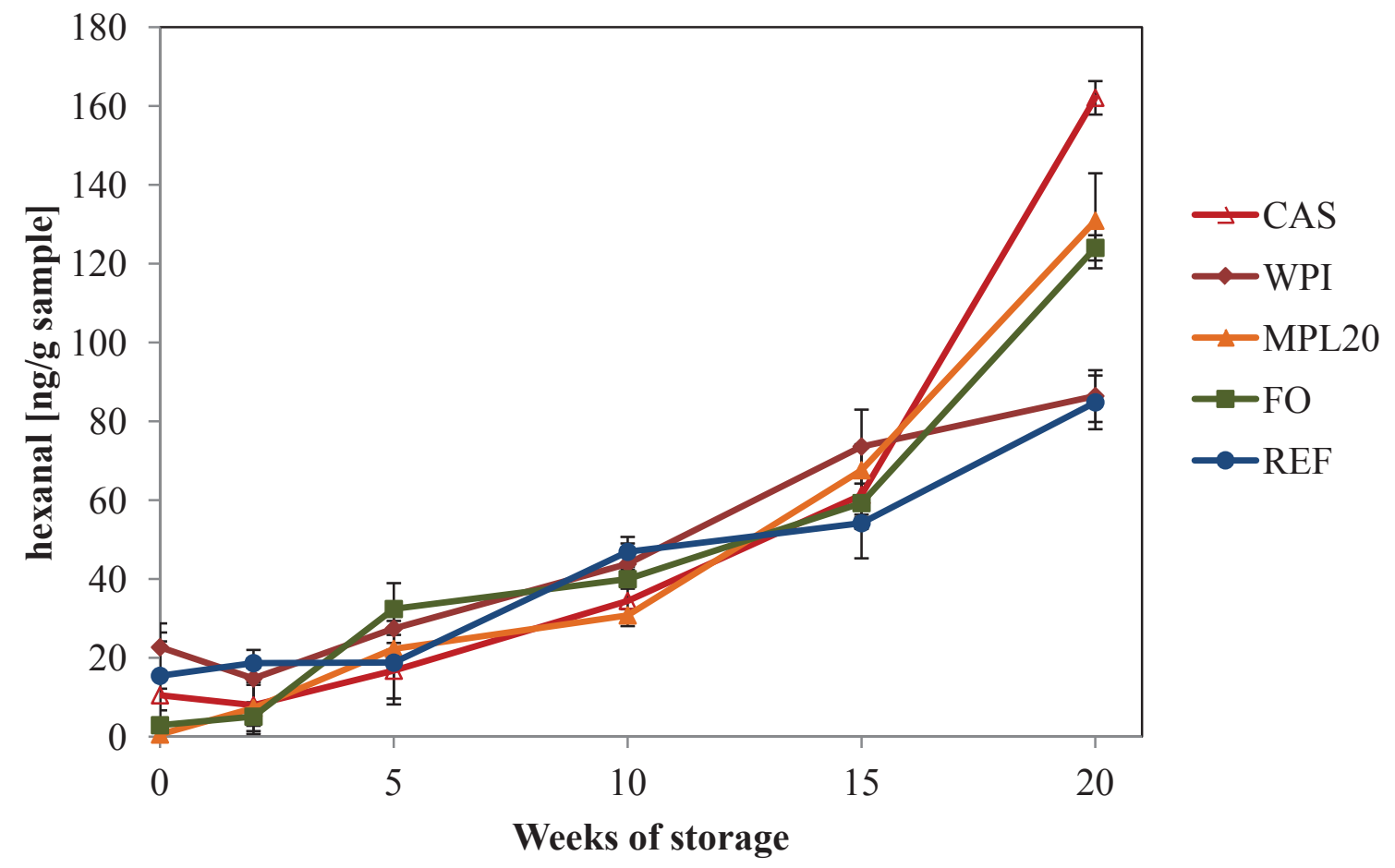

Comparison of individual samples at the different sampling time points showed that until the very last part of the storage period the main differences observed existed between the reference cream cheese and the cheeses added fish oil. However, it was observed that hexanal was the only volatile for which concentrations differed between the samples already at week 0 , with WPI having a higher concentration than all other samples. Concentrations of pentanal and 1-pentanol differed significantly between samples at week 5, and the concentrations of these volatiles were also higher in WPI than any other sample. Concentrations of 2-pentylfurane did not differ significantly between samples until week 10 , where the samples were ranked as follows $\mathrm{REF}^{\mathrm{a}} \leq \mathrm{MPL} 20^{\mathrm{ab}} \leq \mathrm{FO}^{\mathrm{bc}}=\mathrm{CAS}^{\mathrm{bc}} \leq \mathrm{WPI}^{\mathrm{c}}$. Interestingly, at week 20, WPI had in general the lowest concentration of these four volatiles, whereas CAS had the highest. 
The concentrations of the other six group 2 volatiles (butanal, 2-ethylfurane, $t$-2-butenal, $t$-2-hexenal, $t$-2-heptenal and $t, t$-2,4-heptadienal) did not increase significantly between week 0 and 20 in the reference cream cheese, but all increased significantly during storage in the four samples with fish oil added ( $t$-2-hexenal is shown in Figure 4).

Figure 4. The development in $t$-2-hexenal (ng/g sample) in cream cheeses with fish oil added as neat oil (FO), or in a delivery emulsion prepared with different emulsifiers (CAS, WPI or MPL20) compared to a reference cheese without fish oil (REF) during storage for 20 weeks.

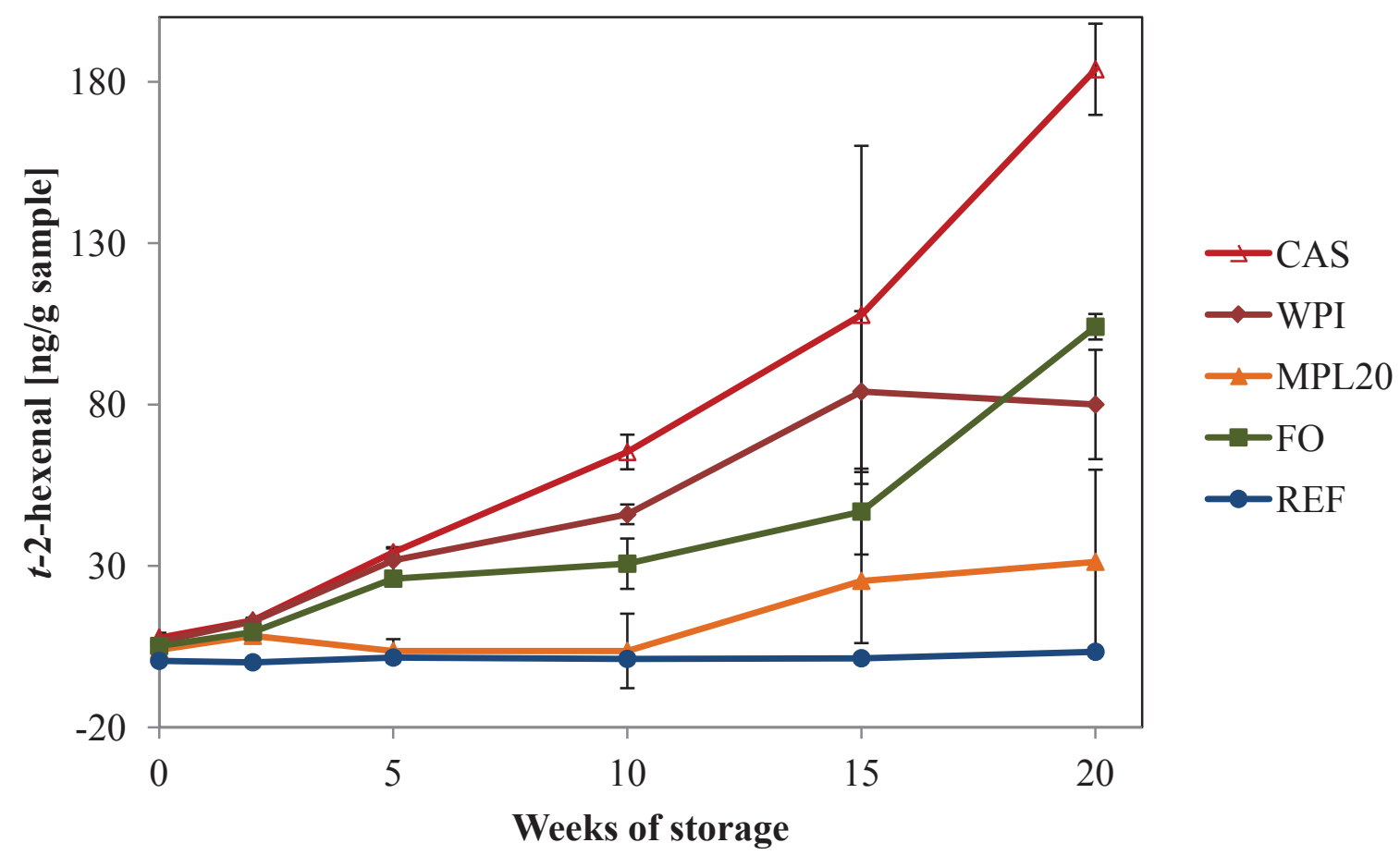

The volatile 2-ethylfuran was one of the first to increase significantly in the fish oil enriched cream cheeses. The concentration increased significantly already between week 2 and 5 in CAS, WPI and FO, and between week 5 and 10 in MPL20. Butanal increased significantly in CAS, WPI and MPL20 between week 5 and 10 whereas FO followed between week 10 and 15 . For the concentrations of the other four volatiles ( $t$-2-butenal, $t$-2-hexenal, $t$-2-heptenal and $t, t$-2,4-heptadienal) a significant increase was not observed in MPL20 until between week 15 and 20. In comparison, a significant increase in $t$-2-butenal and $t$-2-hexenal was observed between week 5 and 10 in CAS and between 10 and 15 in WPI. The FO sample showed more or less a similar pattern to MPL20.

The overall picture from volatiles data was that the reference sample oxidized the least followed by a group of MPL20 and FO and then a group of WPI and CAS. Differences between WPI and CAS were small, but generally CAS oxidized slightly more than WPI. 


\subsubsection{Sensory Profiling of the Cream Cheeses}

Figure 5 shows a PCA model of the results from the sensory profiling. Cream cheeses stored for a short time (all samples at week 2 and 5 and WPI, MPL20 and FO at week 10) had negative scores for PC1 and positive scores for PC2. These cream cheeses were described by salty, sour, sweet and fatty flavors, sour and sweet odors and fatty texture as these descriptors were found at the corresponding location in the correlation loadings plot.

Figure 5. A PCA plot on the data from the sensory profiling of the cream cheeses. The upper figure (A) is the scores plot, and the lower figure (B) is the correlation loadings plot. The first and second principal component explains $76 \%$ and $12 \%$ of the variation, respectively. The numbers given as postfix to the sample names indicate the storage time in weeks.

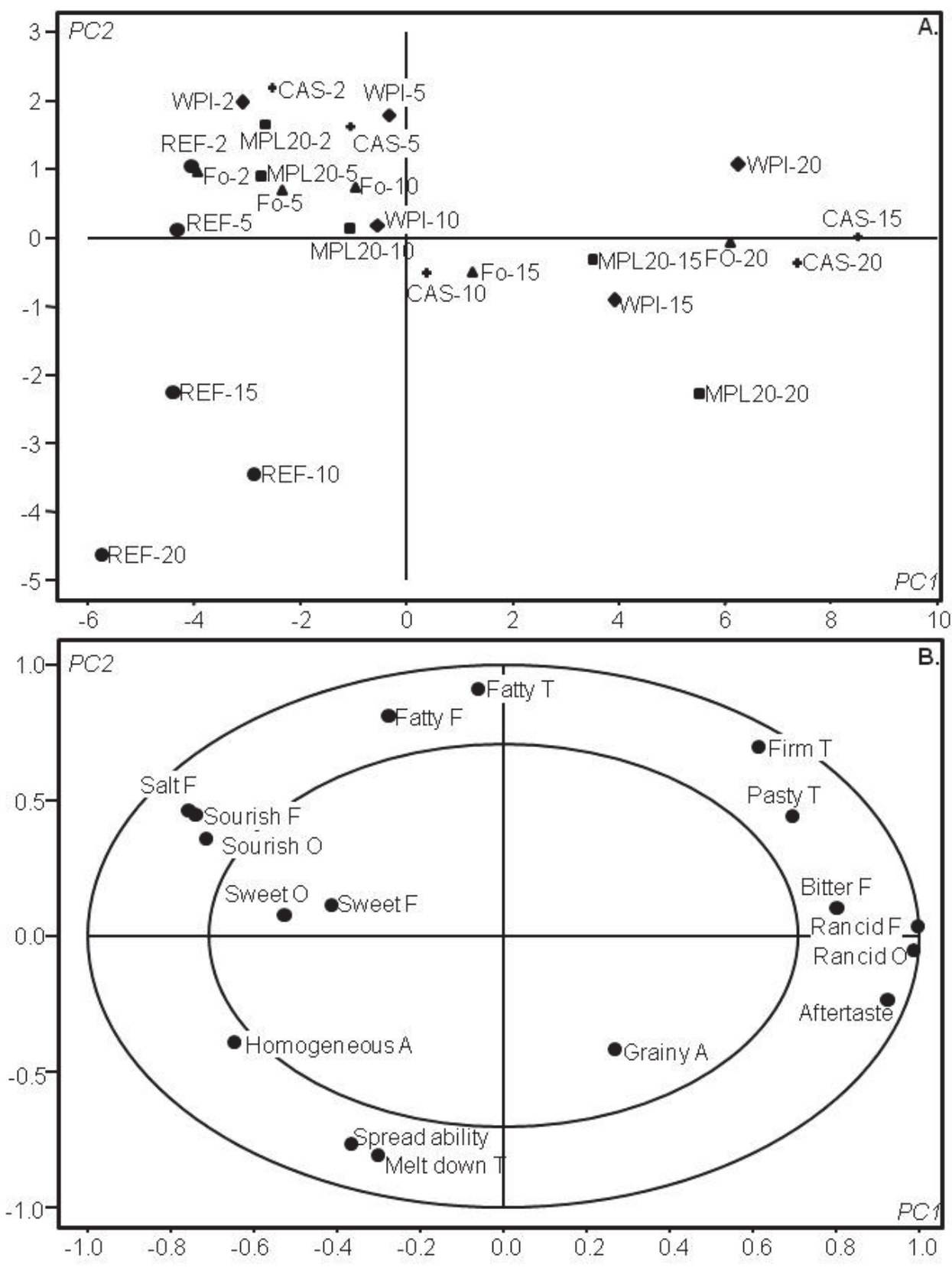


Hence, this finding indicates that the sensory characteristics were generally stable during the first part of the storage period. However, it should be pointed out that especially WPI-5 and CAS-5 had higher PC1 scores than REF-1 indicating that some bitter flavor, aftertaste, rancid odor and flavor had developed in WPI-5 and CAS-5. The intensity of these sensory descriptors increased with storage time for all samples with fish oil, and after 20 weeks of storage all fish oil containing samples were dominated by these characteristics. However, the intensity of these descriptors did not develop equally fast in all the samples. Thus, after 15 weeks, CAS had a PC1 score which was as high as that of the CAS sample that have been stored for 20 weeks. In contrast, none of the other fish oil enriched cream cheeses obtained as high PC1 scores as the CAS samples, even after 20 weeks. This indicates that CAS samples became more rancid than any of the other samples. For the cream cheeses with fish oil-in-water emulsions MPL20, had a lower PC1 score than both WPI and CAS from week 5 and throughout the storage period. Hence, MPL20 developed less bitter flavor and rancid flavor and odor than the other two.

The second principal component mainly explained the variation in the texture and appearance samples. A positive PC2 score, which was seen for most of the samples, was correlated to fatty, firm and pasty texture. In contrast a negative PC2 score was correlated to spreadability, melt down, homogeneous and grainy appearance. Only the reference cream cheese had a negative PC2 score at all sampling points after 5 weeks of storage, whereas the fish oil enriched samples maintained a positive PC2 score throughout storage. Hence, the texture for the reference cream cheese changed from fatty, firm and pasty to a higher degree of homogeneous, grainy, better spreadability and faster melt down but also a more grainy appearance during storage. Apparently, the same changes were not observed in fish oil enriched samples.

\section{Discussion}

Overall, the addition of $1.3 \%(\mathrm{w} / \mathrm{w})$ fish oil to cream cheese increased lipid oxidation during storage as compared to the reference cheese with no fish oil added. In accordance with the study by Kolanowski and Weißrodt (2007) [4], we observed a decrease in the sensory quality after five weeks of storage. Specifically the six volatiles butanal, 2-ethylfurane, $t$-2-butenal, $t$-2-hexenal, $t$-2-heptenal and $t, t-2,4$-heptadienal were observed to increase significantly during storage in the samples with fish oil. Hence, some of these volatiles may have contributed to the development of bitter and rancid off-flavors in the cream cheeses.

In contrast to previous studies where the addition of neat fish oil has been compared to the addition of only one type of delivery emulsion prepared with denatured whey protein in one study or a milk protein complex (casein and whey protein) in another study [2,3], this study investigated three types of delivery emulsions. Hence, despite the unacceptable sensory perception of the fish oil enriched cream cheeses in the later part of the storage period, some interesting observations were obtained on the differences between cheeses. First of all, results showed that the effect of using a delivery emulsion is not straight forward, as the type of delivery emulsion used clearly affected the stability of cream cheeses. Hence, cream cheeses with neat oil or MPL20 emulsion added were found to be similarly oxidized, but less oxidized than cream cheeses with CAS or WPI emulsions added. 
The lack of protecting effect of caseinate was surprising, as casein in a previous study carried out by our research group has been shown to provide the best protection against oxidation on lipid oxidation in simple $70 \%$ fish oil-in-water emulsions (prepared almost similarly to the delivery emulsions added to the cream cheeses but with a $10 \mathrm{mM}$ sodium acetate imidazole buffer instead of water as the aqueous phase) [6]. In addition, the difference between $70 \%$ fish oil-in-water emulsions with sodium caseinate and whey protein isolate were increased when additional iron was added, confirming a metal chelating effect of casein $[6,16]$. In the simple $70 \%$ fish oil-in-water emulsion the antioxidative effect of casein present in the aqueous phase was suggested to be responsible for the good oxidative stability, as also suggested in other studies $[17,18]$. However, this protective effect of casein in the aqueous phase seems to be lacking in the cream cheeses. Hence, metal ions may be chelated by casein surrounding the oil droplets instead whereby metal ions are coming into closer proximity of the oil, than otherwise expected.

With regard to the whey proteins, Let et al. (2007) [2] observed that the oxidative stability of fish oil enriched milk could be increased by adding fish oil as an emulsion after homogenization, and with denatured whey protein as emulsifier. The authors suggested that the advantage of using a delivery emulsion in this system, in contrast to neat oil, was due to a better protection of the fish oil droplets when emulsified by denatured whey protein (in the delivery emulsion) than when emulsified by the protein material present in the milk during homogenization (when neat oil was added). In contrast to milk, the cream cheese undergoes homogenization after addition of fish oil both when it is added as neat oil, and when added as an emulsion, and it is therefore not known exactly which proteins are responsible for emulsifying the fish oil in the cream cheese with the WPI delivery emulsion. However, the protection against oxidation is lower than the protection provided by the protein material present in the cream cheese that emulsifies the fish oil, when it is added as neat oil.

Whereas fish oil enriched milk was shown to be more stable when the oil was added as an emulsion, results from volatiles and sensory data showed that in dressing and yoghurt it was preferable to add the fish oil as neat oil [2]. This finding was explained by the increase in temperature during emulsion production, which led to some oxidation in the emulsion before addition to the food product. This was not compensated for by a better oxidation protection by the emulsion after addition to dressing and yoghurt whereas the opposite was the case for milk. Both milk and cream cheeses are heated during production, but as results on the method of fish oil addition are contradictory for these two food systems, the effect of heating is not unambiguous. In fish oil enriched energy bars, the use of a delivery emulsion prepared with sodium caseinate was observed to increase the oxidative stability of the bars in comparison to the addition of neat fish oil [19]. The increased oxidative stability of energy bars with emulsions added were explained by the ability of caseinate to provide a protective layer around the oil droplets to increase the distance between pro-oxidants in the surrounding matrix. However, a full understanding of why delivery emulsions are successful in some food applications and not in others is still lacking. More knowledge is needed in order to understand the physical structure of the emulsions after addition as well as to understand the interactions between the emulsifier in the delivery emulsion and other ingredients in the food product.

Interestingly, differences in the microstructure of the cream cheeses were also observed between cheeses with fish oil delivery emulsions (WPI, CAS and MPL20) and cheeses without/with neat oil added (REF and FO). These differences may be ascribed to excess protein present in the aqueous phase 
of the $70 \%$ fish oil-in-water delivery emulsions. Hence, when added to the cream cheese, the emulsifier present in the aqueous phase of the delivery emulsion may emulsify the milk fat present in the cream cheese, whereby fewer unprotected milk fat droplets are observed in the micrographs (Figure 1). The emulsification of milk fat by excess casein in the aqueous phase, might therefore also partly explain the lack of protective effect of casein mentioned above.

Differences in the microstructure among cream cheeses with different delivery emulsions was also observed, as even less lipid was visible in the cream cheese with the MPL20 delivery emulsion added than when the other two emulsions (Em_WPI and Em_CAS) were used. The improved incorporation of lipids in the protein structure of the cream cheese with the MPL20 emulsion could help to explain the better oxidative stability of this cream cheese compared to the other two cream cheeses with delivery emulsions added. However, it cannot be the sole explanation as the cream cheese with neat fish oil, where the fish oil is the least incorporated in the protein structure, oxidizes similarly.

More studies are needed, in order to fully understand lipid oxidation in a complex matrix as the cream cheese.

\section{Conclusions and Perspectives}

Overall, the approach of using delivery emulsions for fish oil addition to cream cheese did not improve the oxidative stability of the product during storage, compared to the addition of neat fish oil. Interestingly, the microstructure of the cream cheeses changed substantially when fish oil was added through delivery emulsions. Furthermore, it was observed that the choice of emulsifier for preparing delivery emulsions significantly affects the oxidative stability of the fish oil enriched food product.

To be able to add fish oil to this type of product in the future, and to be able to store the product for more than five weeks, a combination of different approaches for protecting the oxidatively labile fish oil, could be an advantage. This could be the use of a delivery emulsion prepared with a combination of proteins and phospholipids, an optimized protection of the oil during production of the delivery emulsion and the cream cheeses, such as an oxygen-free environment, or the inclusion of antioxidants. Hence, more studies are needed to explore which approach or combination of approaches has the best effect in order to prepare fish oil enriched cream cheese of a good quality with regard to lipid oxidation and the development of off-flavors.

\section{Acknowledgments}

We would like to thank Arla Foods Ingredients amba for donating the emulsifiers, and Maritex A/S, Norway, subsidiary of TINE, BA for providing the fish oil. In addition, we kindly appreciate the help and guidance provided by Kent Matzen and Steffen Øster Jensen during production of the fish oil enriched cream cheeses at Arla Strategic Innovation Centre, Arla Foods amba, and the confocal imaging performed by Betina Mikkelsen. Micrographs were recorded at DTU Center for Electron Nanoscopy, Tecnical University of Denmark, Denmark. Finally, we are grateful for the work carried out by the sensory panel under supervision by Rie Sørensen and Jeannette Møller, and to Lis Berner for her skilful work in the laboratory. This work is part of the project Omega-3 food emulsions: Control and investigation of molecular structure in relation to lipid oxidation. The project is financed by the Danish Food Ministry (DFFE) and DTU globalization funds. 


\section{References}

1. Riediger, N.D.; Othman, R.A.; Suh, M.; Moghadasian, M.H. A systemic review of the roles of n-3 fatty acids in health and disease. J. Am. Diet. Assoc. 2009, 109, 668-679.

2. Let, M.B.; Jacobsen, C.; Meyer, A.S. Lipid oxidation in milk, yoghurt, and salad dressing enriched with neat fish oil or pre-emulsified fish oil. J. Agric. Food Chem. 2007, 55, 7802-7809.

3. Ye, A.; Cui, J.; Taneja, A.; Zhu, X.; Singh, H. Evaluation of processed cheese fortified with fish oil emulsion. Food Res. Int. 2009, 42, 1093-1098.

4. Kolanowski, W.; Weißrodt, J. Sensory quality of dairy products fortified with fish oil. Int. Dairy J. 2007, 17, 1248-1253.

5. EU Commision Regulation No 116/2010. Off. J. Eur. Union 2010, 53, L37:16-L37:18.

6. Horn, A.F.; Nielsen, N.S.; Andersen, U.; Søgaard, L.H.; Horsewell, A.; Jacobsen, C. Oxidative stability of 70\% fish oil-in-water emulsions: Impact of emulsifiers and pH. Eur. J. Lipid Sci. Technol. 2011, 113, 1243-1257.

7. Bligh, E.G.; Dyer, W.J. A rapid method of total lipid extraction and purification. Can. J. Biochem. Physiol. 1959, 37, 911-917.

8. AOCS Official Method Ca 2-66. In Preparation of Methyl Esters of Long Chain Fatty Acids; AOCS: Champaign, IL, USA, 1998.

9. AOCS Official Method Ce 1b-89. In Fatty Acid Composition by GLC, Marine Oils; AOCS: Champaign, IL, USA, 1998.

10. AOCS Official Method Ce 8-89. In Determination of Tocopherols and Tocotrienols in Vegetable Oils and Fats by HPLC; AOCS: Champaign, IL, USA, 1998.

11. Shantha, N.C.; Decker, E.A. Rapid, sensitive, iron-based spectrophotometric methods for determination of peroxide values of food lipids. J. AOAC Int. 1994, 77, 421-424.

12. ISO 1993. In ISO Standards 8586-1. Sensory Analysis-General Guidance for the Selection, Training and Monitoring of Assessors. Part 1. Selected Assessors, 1st ed.; International Standard Organization: Geneva, Switzerland, 1993.

13. ISO 1994. In ISO Standards 11035. Sensory Analysis-Identification and Selection of Descriptors for Establishing a Sensory Profile by a Multidimensional Approach; 1st ed.; International Standard Organization: Geneva, Switzerland, 1994.

14. ISO 1988. In ISO Standards 8589. Sensory Analysis-General Guidance for Design of Test Rooms; 1st ed.; International Standard Organization: Geneva, Switzerland, 1988.

15. Thybo, A.K.; Martens, M. Analysis of sensory assessors in texture profiling of potatoes by multivariate modelling. Food Qual. Prefer. 2000, 11, 283-288.

16. Horn, A.F.; Nielsen, N.S.; Jacobsen, C. Iron-mediated lipid oxidation in 70\% fish oil-in-water emulsions: Effect of emulsifier type and pH. Int. J. Food Sci. Technol. 2012, 47, 1097-1108.

17. Faraji, H.; McClements, D.J.; Decker, E.A. Role of continuous phase protein on the oxidative stability of fish oil-in-water emulsions. J. Agric. Food Chem. 2004, 52, 4558-4564.

18. Ries, D.; Ye, A.; Haisman, D.; Singh, H. Antioxidant properties of caseins and whey proteins in model oil-in-water emulsions. Int. Dairy J. 2010, 20, 72-78.

19. Nielsen, N.S.; Jacobsen, C. Methods for reducing lipid oxidation in fish-oil-enriched energy bars. Int. J. Food Sci. Technol. 2009, 44, 1536-1546. 


\section{Supplementary Material}

Five freeze-fracture cryo-scanning electron micrographs are included as supplementary material. One of each cream cheese.

(C) 2012 by the authors; licensee MDPI, Basel, Switzerland. This article is an open access article distributed under the terms and conditions of the Creative Commons Attribution license (http://creativecommons.org/licenses/by/3.0/). 